\title{
DISTRIBUTED REAL-TIME BASED HUMAN ACTIVITY ANALYSIS SYSTEM
}

\author{
Luigi La Blunda ${ }^{1}$, David Corral-Plaza ${ }^{2}$, Matthias Wagner ${ }^{1}$, Guadalupe Ortiz ${ }^{2}$ \\ and Inmaculada Medina-Bulo ${ }^{2}$ \\ ${ }^{1}$ WSN \& IoT Research Group, Frankfurt University of Applied Sciences \\ Nibelungenplatz 1, 60318 Frankfurt am Main, Hessen, Germany \\ ${ }^{2}$ UCASE Software Engineering Research Group, University of Cadiz. \\ Avda. de la Universidad de Cádiz 10, 11519 Puerto Real, Cádiz, Spain
}

\begin{abstract}
The analysis of physical human activities is essential in order to detect falls in real time and to ensure rapid assistance by the emergency service, especially for people who are exposed to a high risk of falling in everyday life due to dangerous working conditions or certain illnesses. In this scope, we present an ongoing development of a portable motion analysis system that performs continuous movement monitoring regardless of the environment. The system is a real time distributed application consisting of the portable sensor network and an analysis unit based on Stream Processing and Complex Event Processing.
\end{abstract}

\section{KEYWORDS}

Body Area Network, Human Activity Recognition, Stream Processing, Complex Event Processing, Sensor Fusion

\section{INTRODUCTION}

The analysis of human activities concerning hazards is important, especially in the case of falls. In many scenarios of everyday life, people are confronted with the risk of falling. This risk can be drastically increased by working in dangerous conditions, for example, a firefighter who falls during a rescue operation and finds himself in a life-threatening situation. However, it is not only important to act quickly for work-related accidents, but also for older people who have an increased risk of falling, due to certain medical conditions or limited physical mobility. Falls cause older people, who have fallen several times, restrict their movements because they are afraid to fall again. According to this, the World Health Organization (WHO) reports that 37.3 million falls each year cause serious injuries and require medical treatment (WHO, 2018). In order to counteract this life-threatening situation, rapid assistance must be provided because an unconscious person is unable to make the emergency call.

In this scope, we propose a novel portable system that continuously records and analyzes motion activities in order to alert emergency services in case of a fall, without any user intervention. This information flow is based on previously published work (La Blunda, Wagner, 2016a \& La Blunda, Wagner, 2016b). In comparison to the previous publications, the system contains some essential updates which improve the performance and reliability of the prototype.

First of all, the architecture of the prototype has been improved by shrinking the hardware design to provide comfortable portability. This is an important requirement that emerged from the usability tests.

ZigBee was then replaced with Bluetooth Low Energy (BLE), which allows the system to be more flexible in terms of connectivity with other sensors and devices. By using BLE, a smartphone application was developed that allows an efficient connection of the sensors. Besides, the system architecture was restructured into a real-time distributable application, which ensures low latency, compared to the previously published architecture, and fast support in case of a fall event.

The distributed wearable system consists of a Body Area Network (BAN), which is worn on the hip, and an analysis architecture that receives the sensor data from the BAN to provide real time human activity recognition and complex situations detection. 


\section{BASIC TECHNOLOGIES}

\subsection{Wireless Sensor Network}

Wireless Sensor Networks (WSN) are based on wireless connectivity and wireless data transmission. WSNs have a vast applicability area. The special capabilities of wireless communication provide inherent advantages especially for medical applications. Considering our system, wireless connectivity is a crucial feature, as users should be offered a portable and comfortable system without any mobility restrictions. As defined in medical science this fosters patient compliance which is an important aspect for the acceptance of the system.

To build up such a network the following constellation is needed:

- Master / Coordinator: is establishing the WSN and is responsible for the information flow and the pairing of peripherals to the network. Additionally, it is handling data transmission for data evaluation.

- Slaves / Peripherals: are providing sensor data based on a wireless transmission protocol, e.g. ZigBee or Bluetooth Low Energy to the coordinator for analytics. The WSN can include more than one peripheral which leads to a multiple node connection. The possible amount of the connectable nodes depends on the used transmission protocol.

For our proposed BAN-architecture we are using BLE (IEEE 802.15.1), which facilitates a well-organized data structure and multiple possibilities to connect with several devices for data evaluation. The BAN is based on a star topology structure. The used hardware for the BAN consists of the Texas Instruments CC2650 Sensortags.

\subsection{Stream Processing}

Stream Processing (SP) (Vervenica, 2018) is a paradigm for reacting to streams of data as they occur. This technology provides us with the capability of process information in real time, being able to perform transformations over these streams. Using SP we can create distributed and scalable applications for processing data that can be deployed in desktop computers, virtual machines, servers or containers.

There are several solutions for building SP applications such as Apache Storm, Apache Kafka or Apache Flink. For our solution, we have chosen Kafka because it contains the Kafka Streams API, which is specifically designed to create SP applications and it presents some unique advantages against other options such as low latency, fault tolerance, and exactly-once processing semantics.

\subsection{Complex Event Processing}

Complex Event Processing (CEP) (Boubeta-Puig et al., 2015) is a technology that allows us to detect complex situations by analyzing data that come as simple events. These simple events are readings acquired from data sources such as IoT sensors (in this work accelerometers and gyroscopes), database rows, third-party APIs, et cetera. The alerts (complex events) to be detected have to be specified in patterns, which are composed of certain conditions that these simple events have to satisfy to trigger the pattern up. The structure and syntax of these patterns will depend on the CEP solution to use.

Among the different solutions existing for CEP, we would like to highlight Apache Flink and Esper. Flink can be used for more purposes than just CEP, while Esper has been specifically created for that. In Flink, you can create some SP process and then perform analytics over these streams of data meanwhile in Esper the data is directly analyzed. Esper has a specific SQL-similar syntax, Event Pattern Language (EPL) which provides us with any kind of stream operations such as data windows, filters, operators, built-in functions, and so on. 


\section{USE CASE AND PROPOSED ARCHITECTURE}

\subsection{Human Activity Recognition}

The presented BAN consists of a four-node sensor belt, each node with 3-axis accelerometers and gyroscopes, which creates a multiple node connection with an iOS prototype application and sends continuous data to determine in real time the actual human activity and in case of a fall event to contact immediately the emergency services. The smartphone application is acting as the master device and it should facilitate multiple simultaneous connections. Since we are developing a safety critical system, functional safety must be ensured in order to prevent human life from being endangered. Therefore, 4 nodes are positioned symmetrically to ensure reliable detection even if one node fails.

\subsection{Proposed Architecture}

In Figure 1 the proposed architecture is shown. It is based on a previous architecture shown in (Corral-Plaza et al., 2018). This architecture is structured in 3 layers:

- Data Producers: this layer is in charge of producing the information that is going to be analyzed. For our scenario, the data producers will be the four sensors that are measuring, the information relative to the person's posture. In addition, in this layer, we have implemented an iOS application which is in charge of collecting the information from the sensors and send it to the Data Processing layer.

- Data Processing: this layer is responsible of sensor information processing and analyzing, in real time, which is received as CSV rows.

- Data Consumers: this layer is composed of any kind of smart device which will consume the alerts which are detected by the Esper CEP Engine. These are, for example, web applications, databases, smartphones, et cetera.

DATA PRODUCERS

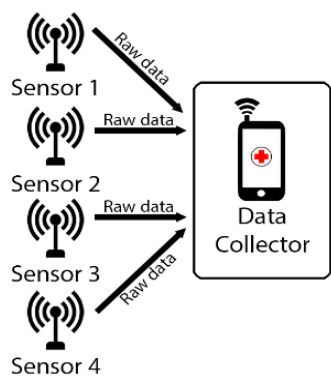

DATA PROCESSING

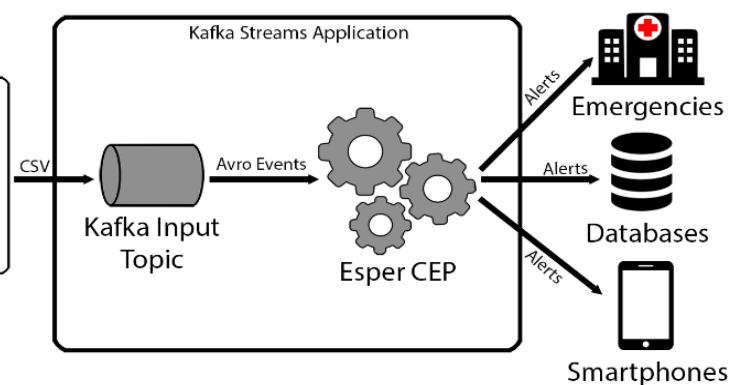

Figure 1. Proposed system architecture

The data producers layer is composed of IoT sensors which are generating information through observations from the real world. In the fall detection scenario, which we are implementing, this layer will be composed of sensors which are measuring information related to the posture of a person. These events are collected in an iOS App which is in charge of synchronizing these sensors, retrieving the information and publishing it into a Kafka Input topic.

The data processing layer is in charge of receiving the data, processing them, and analyzing them. These tasks are achieved using Kafka Streams API along with Esper. At first, the data is received in a Kafka Input topic. Once a message is received in the topic, it is consumed by the Kafka Stream Application, transformed into the suitable format, and sent to the Esper CEP engine to be analyzed. Subsequently, the simple events that are received in the Esper CEP engine will be evaluated against the EPL patterns. When all the conditions specified in the pattern are satisfied, a new complex event (situation of interest) is triggered and will be notified to the Data Consumers layer. In our case, these complex events that have to be detected are the postures of the person which are as follows: standing, sitting, laying on back, laying on the stomach, laying on the left, and laying on the right. 
Finally, once the complex events are triggered by the Esper CEP engine, they are pushed to the data consumers layer, which can be composed of emergencies services, databases, smartphones, et cetera.

\section{EVALUATION}

In order to prove the effectiveness of the proposed solution, we have performed practical experiments in cooperation with the Fire Brigade of Frankfurt am Main (Feuerwehr Frankfurt am Main, 2019). In these experiments, the four sensors are generating data every 25 milliseconds, which means that in a second an amount of 40 events are generated per sensor, having 4 sensors results in a total of 160 events per second.

These events are collected by the iOS application (Data Collector) and are published in the Kafka Input topic. The Kafka Stream Application consumes them, prepares the simple events, and sends them to the Esper CEP engine, where they are analyzed. Once in the Esper CEP engine, these events are evaluated against the patterns, detecting the situations of interest in real time when the conditions of these patterns are satisfied.

These patterns are based on physical models which depict the posture of a person. Considering the orientation of the individual nodes in relation to the room coordinate system, the gravitational force of $1 \mathrm{G}$ acts on the $\mathrm{X}$-axis of the accelerometer of all four sensor nodes in a standing or sitting person. In this way, Formula 1 illustrates the physical equation for standing or sitting posture using four sensor nodes, while Listing 1 shows the EPL pattern implementation. First, we name the pattern using the keyword '@NAME'. Second, we define the list of attributes to be projected using 'SELECT' keyword followed by these attributes. Third, we specify the simple event types to be used as a source, in this case four sliding windows, one for each sensor, of 'HumanActivity', with the keyword 'FROM'. Finally, 'WHERE' is used to specify the conditions that the simple events previously defined have to satisfy in order to generate this complex event.

$$
\begin{gathered}
0.75[\mathrm{G}] \leq \mathrm{a}_{\mathrm{xS} 1} \leq 1.25[\mathrm{G}] \& 0.75[\mathrm{G}] \leq \mathrm{a}_{\mathrm{xS} 2} \leq 1.25[\mathrm{G}] \& 0.75[\mathrm{G}] \leq \mathrm{a}_{\mathrm{xS} 3} \leq 1.25[\mathrm{G}] \\
\& 0.75[\mathrm{G}] \leq \mathrm{a}_{\mathrm{xS} 4} \leq 1.25[\mathrm{G}] \\
\mathrm{a}_{\mathrm{xS} 1}, \mathrm{a}_{\mathrm{xS} 2}, \mathrm{a}_{\mathrm{xS} 3}, \mathrm{a}_{\mathrm{xS} 4} \rightarrow \mathrm{x} \text {-Acceleration in }[\mathrm{G}] \text { for sensor node } 1 \text { to } 4 \\
1 \mathrm{G} \approx 9.81 \mathrm{~m} / \mathrm{s}^{2}
\end{gathered}
$$

Formula 1. Physical model for standing or sitting posture

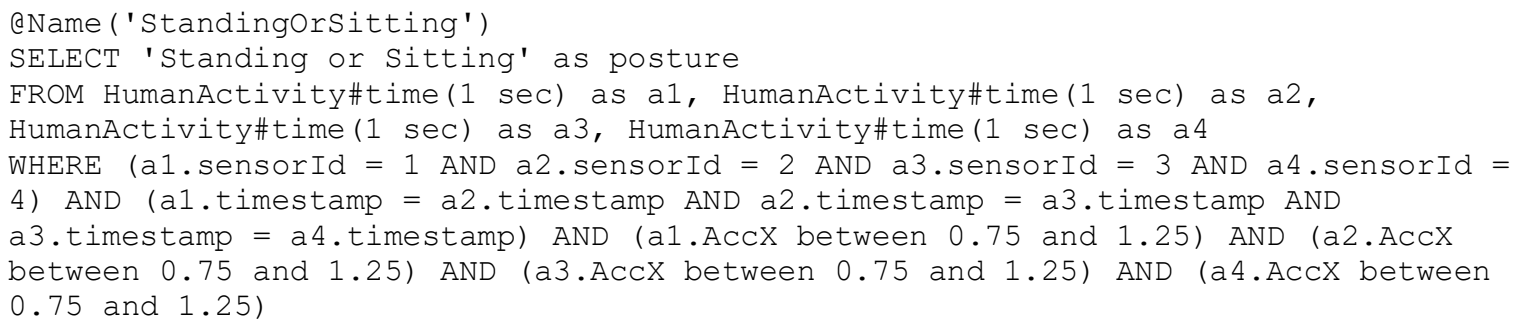

Listing 1. EPL implementation for standing or sitting posture

Finally, when the complex event is triggered, it is sent to the Data Consumers, which for testing purposes right now is just a Kafka Output topic that is used in order to visualize these complex situations detected.

The evaluations performed were successful, as the architecture was able to detect, in real time, the six postures (standing, sitting, laying on back, laying on the stomach, laying on the left, and laying on the right) even when they changed.

\section{CONCLUSIONS AND FUTURE WORK}

In this work, we have presented a distributed processing system for monitoring people's posture. The main advantage of this system is the capability to perform analytics in real time, being able to detect falls or accidents as soon as possible. The system is currently able to detect reliably the postures mentioned above in a test situation. As future work, we are planning to increase the number of sensors, integrate them in the architecture 
and analyze more data sources in real time, with the aim of providing more precise results. In addition, the integration of machine learning techniques in order to facilitate fall prediction is prepared. Finally, the development of the Android app to retrieve the information of the sensors is planned.

\section{ACKNOWLEDGEMENT}

This work was supported in part by the Spanish Ministry of Science and Innovation and the European Union FEDER Funds (grant number TIN2015-65845-C3-3-R and RTI2018-093608-B-C33) and partly by the pre-doctoral program of the University of Cádiz (2017-020/PU/EPIF-FPI-CT/CP).

\section{REFERENCES}

Boubeta-Puig, J., Ortiz, G., \& Medina-Bulo, I., 2015. MEdit4CEP: A model-driven solution for real-time decision making in SOA 2.0. Knowledge-Based Systems. 89, 97-112.

Corral-Plaza, D., Medina-Bulo, I., Ortiz, G., \& Boubeta-Puig, J., 2018, Hacia una arquitectura para el procesamiento y análisis en tiempo real de datos heterogéneos en IoT. Actas de las XIV Jornadas de Ciencia e Ingeniería de Servicios. Presented at the JCIS 2018. Sevilla, España. https://doi.org/hdl.handle.net/11705/JCIS/2018/012.

Feuerwehr Frankfurt am Main, 2019, FRTC. http://www.feuerwehr-frankfurt.de/frtc/ Last access: 29-07-2019.

La Blunda, L. and Wagner, M., 2016, Fall-detection belt based on body area networks. Proceedings of Biotelemetry XXI ISOB conference. Leuven, Belgium, vol. 1, pp. 21-24.

La Blunda, L. and Wagner, M, 2016, The Usage of Body Area Networks for Fall-Detection. Proceedings of the eleventh International Network Conference (INC 2016). Frankfurt am Main, Germany, pp. 159-163.

Vervenica, 2018, What is Stream Processing. https://www.ververica.com/what-is-stream-processing Last access: 29-07-2019.

WHO, 2018, Falls. https://www.who.int/news-room/fact-sheets/detail/falls Last access: 25-06-2019. 